\title{
Spectral observations of transient features in the FUV Jovian polar aurora
}

\author{
J.-C. Gérard, J. Gustin, and D. Grodent \\ Laboratoire de Physique Atmosphérique et Planétaire, Institut d'Astrophysique et de Geophysique, Université de Liége, \\ Liege, Belgium \\ J. T. Clarke \\ Center for Space Physics, Boston University, Boston, Massachusetts, USA
}

\section{A. Grard}

Laboratoire de Physique Atmosphérique et Planétaire, Institut d'Astrophysique et de Geophysique, Université de Liége, Liege, Belgium

Received 17 February 2003; revised 2 May 2003; accepted 23 May 2003; published 13 August 2003.

[1] Images of the Jovian FUV aurora show several morphologically and dynamically different regions. The main oval is usually fairly steady while the high-latitude emission inside the oval can vary over timescales of $<1 \mathrm{~min}$. We describe time and spatially resolved spectral observations of the Jovian aurora made with the Space Telescope Imaging Spectrograph on board the Hubble Space Telescope. Time-tagged spectra have been used to determine variations of the amount of hydrocarbon absorption above the auroral source layer. Rapid brightenings are observed in the high-latitude emission with typical lengths of 50-100 s. They generally are not correlated with enhancements of the main oval in the same longitude sector, indicating decoupled source regions in the magnetosphere. The location of the high-latitude spots and their variability suggest that, unlike the main auroral oval, the polar cap brightenings are connected to short-term variations of the solar wind parameters. Results from a two-stream electron transport model are used to convert observed spectral color ratios into mean electron energies $\langle E\rangle$. Electron energies during these brightenings typically range from 40 to $120 \mathrm{keV}$, close to the values found in the main oval. The time evolution of $\langle E\rangle$ generally shows little correlation or anticorrelation with the energy flux precipitated during these transient events. This feature suggests that the mechanism responsible for rapid transient brightenings does not increase the energy of the precipitated electrons, but it enhances their number flux. Pitch angle scattering into the loss cone by magnetic pumping of energy perpendicular to the field lines following magnetic field compression is a possible process. INDEX TERMS: 6220 Planetology: Solar System Objects: Jupiter; 2704 Magnetospheric Physics: Auroral phenomena (2407); 2716 Magnetospheric Physics: Energetic particles, precipitating; KEYWORDS: Jovian aurora, polar flares, time variation, electron precipitation

Citation: Gérard, J.-C., J. Gustin, D. Grodent, J. T. Clarke, and A. Grard, Spectral observations of transient features in the FUV Jovian polar aurora, J. Geophys. Res., 108(A8), 1319, doi:10.1029/2003JA009901, 2003.

\section{Introduction}

[2] The Jovian aurora was first imaged with the far ultraviolet cameras (Faint Object Camera, Wide Field and Planetary Camera 2, and Space Telescope Imaging Spectrograph (STIS)) on board the Hubble Space Telescope (HST). Its morphology may be globally characterized [Gérard et al., 1994; Ballester et al., 1996; Grodent et al., 1997; Prangé et al., 1998; Clarke et al., 1996, 1998] by (1) a main auroral oval which magnetically maps in the magnetosphere beyond $20 R_{J}$, (2) diffuse emission scattered

Copyright 2003 by the American Geophysical Union. 0148-0227/03/2003JA009901\$09.00 inside the main oval showing large time variations on timescales of minutes, and (3) an Io flux tube footprint spot with a trailing tail sometimes extending over nearly $180^{\circ}$ of longitude. On the morningside, the main auroral oval is defined by a bright arc which may be enhanced near the dawn limb during occasional storms. In the afternoon the main oval is generally more diffuse and weaker than in the morning sector.

[3] The main oval maps to $\sim 20-30 R_{J}$, a region beyond which departure from corotation becomes increasingly significant. Cowley and Bunce [2001] and Hill [2001] proposed a picture where the main oval precipitation is related to the magnetosphere-ionosphere current circuit associated with corotation enforcement at distances of 
20-30 $R_{J}$. The radial current within the plasma sheet closes in the ionosphere via field-aligned currents associated with departures of the plasma motion from corotation. Bunce and Cowley [2001] suggested that large fieldaligned potential drops of $\sim 50-150 \mathrm{keV}$ must exist along middle magnetosphere field lines to drive the upward currents carried by precipitating magnetospheric electrons. This energetic electron precipitation is associated with the main oval aurora.

[4] In situ plasma measurements of energetic electrons traveling along field lines connected to the Jovian aurora have been made only at large distances from the ionosphere. Field-aligned beams of electrons of energies above $50 \mathrm{keV}$ were detected on board the Ulysses spacecraft at radial distances from 15 to $75 R_{J}$. Impulsive bursts of relativistic electrons were observed on the dusk side, sometimes as isolated events but at other times as quasiperiodic series with a period of $\sim 40 \mathrm{~min}$. [McKibben et al., 1993]. For smaller events, shorter periods ( $\sim 2 \mathrm{~min})$ were also occasionally observed. At $25 R_{J}$ the Ulysses spacecraft crossed magnetic field lines intercepting the Jovian ionosphere at higher latitudes than the main oval. These measurements are thus associated with the high-latitude emission inside the main auroral oval described by Clarke et al. [1996] and also discussed in this study. Frank and Paterson [2002] presented measurements carried out with the plasma instrumentation (PLS) on board the Galileo spacecraft between 9 and $50 R_{J}$ in the morning and premidnight magnetosphere. Intense electron beams narrowly collimated parallel and antiparallel to the magnetic field were measured at distances of $\sim 20$ to $30 R_{J}$ and identified with the main auroral oval. Their energies ranged from several $\mathrm{keV}$ to $100 \mathrm{keV}$, and the energy flux they carried extended up to $\sim 100 \mathrm{~mW} \mathrm{~m}^{-2}$. The presence of intense antiparallel beams near the equatorial plane is not in agreement with auroral electrons accelerated by parallel electric fields as postulated by Cowley and Bunce [2001] and Hill [2001]. Frank and Paterson [2002] attributed the source of the electron beams to intense plasma heating at low altitudes.

[5] High-latitude spatially structured FUV emissions are frequently observed poleward of the main oval, especially in the noon and afternoon sectors of the planet. Their location and brightness can vary rapidly as evidenced by changes observed in HST images taken minutes apart [Clarke et al., 1998; Grodent et al., 2003]. Very dynamical and intense high-latitude emissions ("polar flares") were reported by Waite et al. [2001] from time-tagged STIS images. These transient brightenings are characterized by a considerable increase in size and brightness over periods of $10-100 \mathrm{~s}$. In one case, the precipitated energy flux locally exceeded $3 \mathrm{Wm}^{-2}$ at the peak of the event. Gladstone et al. [2002] observed a pulsating X-ray "hot spot" inside the main auroral oval. This spot was fixed in magnetic latitude and longitude and pulsated with a 45-min period.

[6] Baron et al. [1996] showed the existence of a dayto-day relationship between the total $\mathrm{H}_{3}^{+}$auroral brightness and the solar wind ram pressure. Moderate brightness variations were also observed over $\sim 100 \mathrm{~s}$ with the ultraviolet spectrometer (UVS) on Galileo [Pryor et al., 2001]. However, lack of spatial resolution did not permit the contribution of the main auroral oval to be separated from the high-latitude emission. Similarly, changes in the power of hectometric radio emissions originating from the Jovian aurora are controlled by the solar wind [Desch and Barrow, 1984; Gurnett et al., 2002]. The available evidence therefore suggests that the dynamics of the high-latitude features is controlled by solar wind interaction with the distant Jovian magnetotail and dayside magnetopause.

[7] STIS-HST spatially resolved spectroscopy offers an opportunity to simultaneously measure both the auroral brightness and the FUV color ratio in different locations of the Jovian aurora. The FUV color ratio $(\mathrm{CR}) \mathrm{CR}=$ $[I(1550-1620 \AA) / I(1230-1300 \AA)]$ provides a measure of the attenuation of the $\mathrm{H}_{2}$ emission below $1350 \AA$ by hydrocarbons, mainly methane, overlying the emission layer (Figure 1). It depends on the vertical distribution of the emission with respect to the FUV absorbers and is therefore an indicator of the amount of absorption by hydrocarbon above the emitting layer. Coupled with an electron transport model, the color ratio may be used to determine the characteristic energy of the precipitation [Yung et al., 1982]. Spatially unresolved FUV spectroscopy of the Jovian aurora was used to determine globally averaged color ratio with the International Ultraviolet Explorer (IUE), the Hopkins Ultraviolet Telescope (HUT), and the UVS spectrometer on board the Galileo spacecraft [Livengood et al., 1990; Harris et al., 1996; Morrissey et al., 1997; Ajello et al., 1998]. Some spatial resolution became available with the Goddard High Resolution Spectrometer (GHRS) on board HST which intercepted a $2 \times 2 \operatorname{arc~sec}^{2}$ sector on the planet [Trafton et al., 1994; Kim et al., 1997]. The results indicated that the overlying $\mathrm{CH}_{4}$ column density varies between 1 and $7 \times 10^{16} \mathrm{~cm}^{-2}$. Dols et al. [2000] analyzed seven auroral GHRS spectra when the GHRS aperture was moved to different latitudes and local times. Large variations of the methane slant column density from 2.3 to $70 \times 10^{16} \mathrm{~cm}^{-2}$ were found, reflecting both the changes of the view angle and variations of the mean electron energy between 35 and $80 \mathrm{keV}$.

[8] In this study, we combine the advantages of the spatial resolution of STIS long slit, the wide spectral coverage of the G140L grating and the time tag mode to investigate the characteristics of the fast transient brightenings occasionally observed in the Jovian high-latitude aurora. In particular, we analyze the energy-flux relationship during these events to determine whether they are induced by the buildup of a parallel electric field energizing electrons or to an increased amount of electron precipitation at roughly the same mean energy.

\section{Observations and Model}

[9] The spatially resolved spectra were imaged on the FUV MultiAnode Microchannel Array (MAMA) photon counting detector providing a $1024 \times 1024$ pixel image. The G140L grating combined with the $52 \times 0.5$ or the $52 \times$ $0.2 \operatorname{arc~sec}^{2}$ long slit provides spectra in the $1100-1700 \AA$ range with $\sim 12$ - or $4.8-\AA$ resolution. The instrument was operated in the TIMETAG mode and provided an event stream with fast time resolution. Processing of the STIS spectra and the background subtraction were described by 


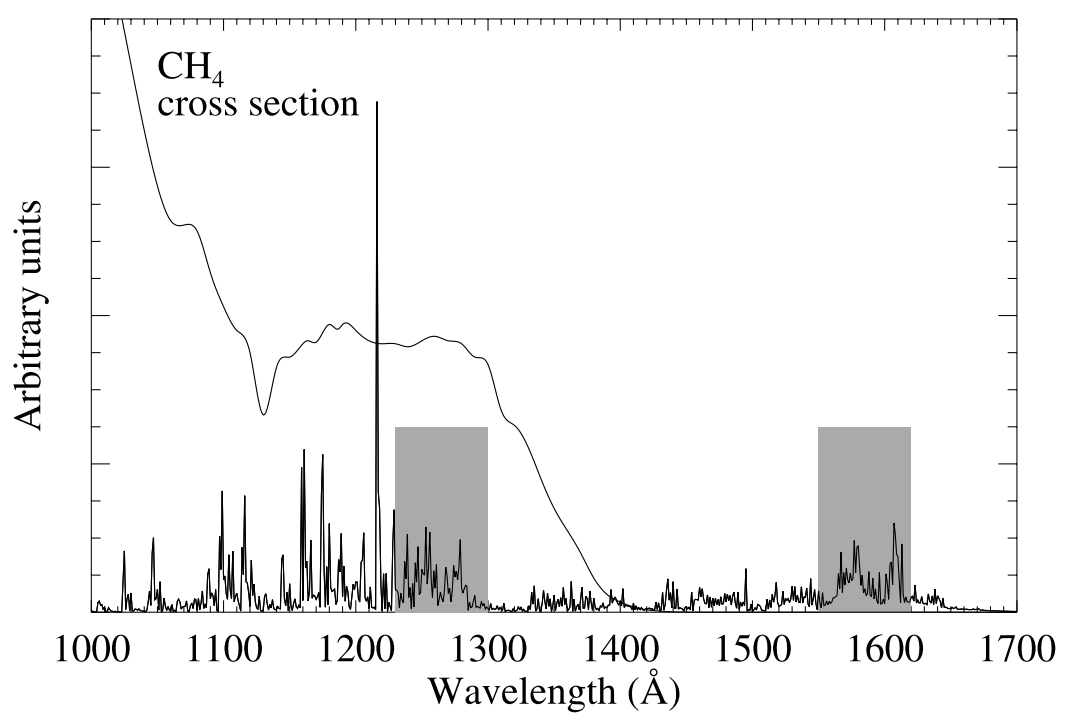

Figure 1. Synthetic unabsorbed $\mathrm{H}_{2}$ spectrum for $T=500 \mathrm{~K}$, including Lyman- $\alpha$ and variation of the methane absorption cross section with wavelength. The intensity units are proportional to kiloRayleighs per angstrom $\left(\mathrm{kR}^{-1}\right)$. The ratio of the brightness of the two shaded areas depends on the amount of hydrocarbon absorption and provides the FUV color ratio.

Gustin et al. [2002]. However, for the G140L spectra used in this study and in the two spectral windows defining the color ratio, the "dark blotch" is much less important than in the weak 1300- $\AA$ region described by Gustin et al. [2002]. The high-latitude regions discussed in this study are all in the sunlit part of the Jovian disk. However, in these highlatitude emissions, the dayglow and solar Rayleigh scattered contributions in the two spectral windows used in the CR are small in comparison with the auroral signal $(<10 \%)$. The count rate is converted into physical units of kiloRayleighs $(\mathrm{kR})$ and $\mathrm{mW} \mathrm{m}^{-2}$ (equivalent to erg $\mathrm{cm}^{-2} \mathrm{~s}^{-1}$ ), based on the absolute instrumental response, in-flight calibration and synthetic $\mathrm{H}_{2}$ spectra.
[10] Table 1 lists the observation dates and times of the spectra described in details in this study, together with the exposure time, hemisphere, central meridian, and approximate position relative to the central meridian (CML). A summary plot of the complete data set of auroral STIS spectra obtained between July 1997 and January 2001 was presented by Gérard et al. [2002]. Gerard et al.'s Figure 2 showed the variation of the color ratio as a function of dates for three different latitudinal regions of the Jovian aurora. The color ratio of the auroral main oval, polar cap, and Io tail exhibits distinct behaviors. The main oval shows large variations ranging from 1.4 to 10.4 and above. The polar cap ratio is also highly variable, although no values higher

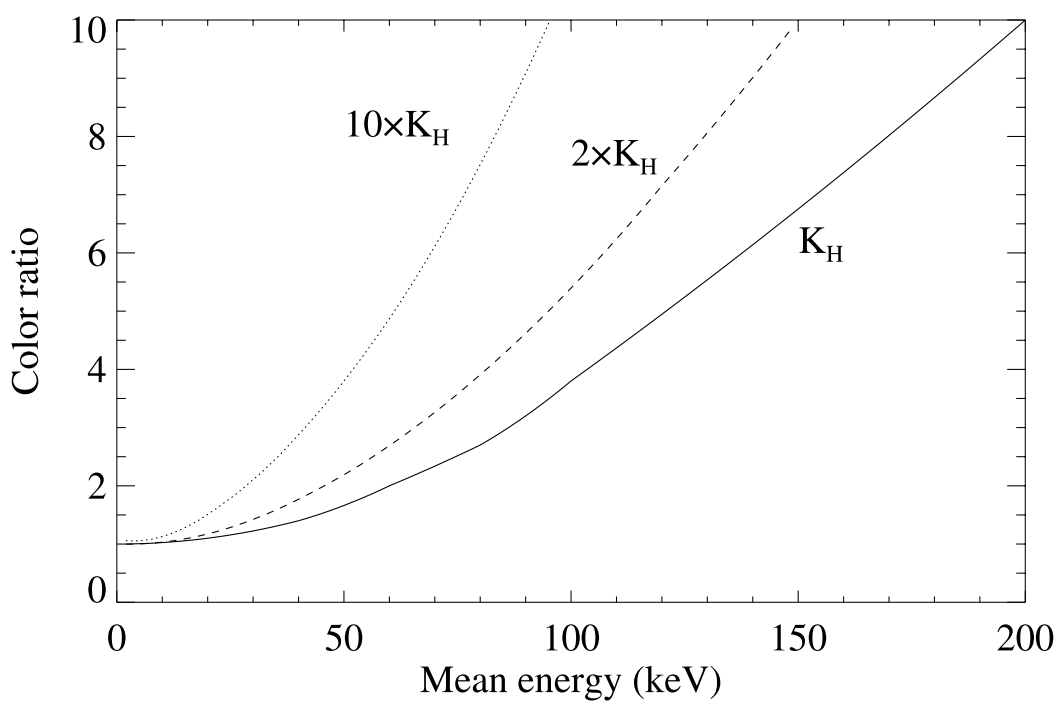

Figure 2. Calculated variation of the FUV color ratio as a function of the mean electron energy for a Maxwellian distribution of the primary electrons at the top of the atmosphere and a view angle of $60^{\circ}$. The three curves illustrate the sensitivity of the relationship to vertical eddy transport for eddy diffusion coefficients $K_{H}=1.4 \times 10^{6}, 2.8 \times 10^{6}$, and $1.4 \times 10^{7} \mathrm{~cm}^{2} \mathrm{~s}^{-1}$ at the homopause. 
Table 1. Time Tag Space Telescope Imaging Spectrograph Spectral Observations Described in This Study ${ }^{\mathrm{a}}$

\begin{tabular}{ccccccc}
\hline Obs. & Date & Time, UT & Exposure, $\mathrm{s}$ & $\mathrm{N} / \mathrm{S}$ & Central Meridian Longitude (SIII), deg. & Aperture \\
\hline 1 & 14 Dec. 2000 & 1213 & 100 & $\mathrm{~N}$ & 210 & MAMA \\
2 & 14 Dec. 2000 & 1221 & 630 & $\mathrm{~N}$ & 219 & $52 \times 0.5 \mathrm{arc} \mathrm{sec}^{2}$ \\
3 & 20 Jan. 2001 & 1550 & 108 & $\mathrm{~N}$ & 156 & MAMA \\
4 & 20 Jan. 2001 & 1608 & 225 & $\mathrm{~N}$ & 53 & $52 \times 0.5 \mathrm{arc} \mathrm{sec}^{2}$ \\
5 & 4 Jul. 1997 & 0931 & 700 & $\mathrm{~S}$ & 63 & $52 \times 0.5 \mathrm{arc} \mathrm{sec}^{2}$ \\
6 & 4 Jul. 1997 & 0947 & 1400 & $\mathrm{~S}$ & $52 \times 0.2 \mathrm{arc} \mathrm{sec}^{2}$ \\
\hline
\end{tabular}

${ }^{a}$ Obs., observation; N, north; S, south; MAMA, MultiAnode Microchannel Array.

than 6.7 are observed. In comparison, the Io trail color ratio is nearly constant and low in comparison with the other regions.

[11] Using an atmospheric model of the Jovian atmosphere, the FUV auroral color ratio may be used to determine the mean energy of the precipitated electrons. The model to calculate the interaction of the energetic electrons with the Jovian atmosphere was described in detail by Grodent et al. [2001]. In the version used for this study the thermal structure is not allowed to adjust to the local thermal balance. The neutral atmosphere is taken from Gladstone et al.'s [1996] North Equatorial Belt model. The gravity acceleration is adapted to latitude of $60^{\circ} \mathrm{N}$. The methane vertical distribution is obtained by combining the lower boundary mixing ratio from Drossart et al. [1993] with the solution of the one-dimensional diffusion equation. The eddy coefficient is set to $1.4 \times 10^{6} \mathrm{~cm}^{2} \mathrm{~s}^{-1}$ at the homopause. Synthetic $\mathrm{H}_{2}$ spectra are generated as described by Dols et al. [2000]. The emerging intensity of each $\mathrm{H}_{2}$ line is calculated from the weighted sum of the spectra, including hydrocarbon absorption when present. The final emerging spectrum is smoothed by the instrumental line spread function and the CR is calculated for a given initial electron energy spectrum. Since the electron energy spectrum incident associated with polar brightenings has not been well characterized by in situ observations, we use a Maxwellian energy distribution defined by its mean electron energy $\langle\mathrm{E}\rangle=2 E_{0}$ and number flux $N_{0}$ and having an energy flux $F_{0}=2 N_{0} E_{0}$, where $\mathrm{E}_{0}$ is the characteristic energy. The calculated relationship between the color ratio and $\langle E\rangle$ for a view angle of $60^{\circ}$ is shown in Figure 2 . The sensitivity of the electron energy determination to the value of the eddy diffusion coefficient, $K$, is also shown by varying the value of $K$ at the homopause by factors of 2 and 10. Analysis of a star occultation by the polar Jovian atmosphere provided a value of $3.5 \times 10^{6} \mathrm{~cm}^{2} \mathrm{~s}^{-1}, \sim 2.5$ higher than our standard value [Raynaud et al., 2003]. In this case, a given color ratio corresponds to a lower electron energy than if the standard $K$ value is used. For example, a typical CR of 4 in the main oval translates into $105 \mathrm{keV}$ for $K=1.4 \times 10^{6} \mathrm{~cm}^{2} \mathrm{~s}^{-1}$ and $80 \mathrm{keV}$ for a doubling of $K$. This uncertainty must be kept in mind when converting color ratios into electron characteristic energies.

[12] The $\mathrm{H}_{2}$ brightness used in this paper refers to the total emission between 1140 and $1700 \AA$, which contributes $69 \%$ of the Lyman $(\mathrm{B} \rightarrow \mathrm{X})$ and Werner $(\mathrm{C} \rightarrow \mathrm{X})$ emissions and $62 \%$ of the total $\mathrm{H}_{2}$ ultraviolet excitation (including other singlet Rydberg states). These values are based on a detailed model [Gustin et al., 2002] of energetic electron transport and energy loss. However, self-absorption becomes increasingly important below $\sim 1200 \AA$, leading to attenuation of the EUV component and partial redistri- bution of photons toward longer wavelengths with an efficiency depending on the amount of overlying $\mathrm{H}_{2}$ molecules and their temperature. Previous studies [Gérard and Singh, 1982; Waite et al., 1983; Grodent et al., 2001] indicate that the efficiency of the electron energy conversion into $\mathrm{B}$ and $\mathrm{C}$ states excitation is close to $10 \mathrm{kR} /$ incident $\mathrm{mW} \mathrm{m} \mathrm{m}^{-2}$ for primary energies between 10 and $100 \mathrm{keV}$.

\section{Time Variations}

[13] The time tag mode of the STIS instrument makes it possible to observe spatially resolved spectral variations over periods of several minutes. The first spectrum we describe was preceded by the STIS image in the MAMA clear mode transmitting FUV light from 1150 to $\sim 1750 \AA$. Figure 3a shows the STIS image obtained on 14 December 2000 at $1213 \mathrm{UT}, 8 \mathrm{~min}$ before the first spectrum. A complete main oval is seen encircling the magnetic pole. Some diffuse emission is observed equatorward of the oval, and the Io footprint with its duskward trailing tail is clearly apparent. A secondary arc is present inside the main oval in the afternoon sector. Patchy emission is also scattered inside the oval both in the morning and afternoon sectors. As discussed and illustrated by Gérard et al. [2002], the projection of the $52 \times 0.5 \operatorname{arc~sec}^{2}$ slit is first overlaid on the image taken closest in time before or after each spectrum. The disk is entirely in daylight with the exception of a small $\left(3.6^{\circ}\right)$ crescent along the dawn terminator.

[14] During the $8 \mathrm{~min}$ separating the image from the spectrum, the bulk of the auroral emission rotates by $\sim 5^{\circ}$ along parallels. In the region considered this motion is of no consequence on the slit positioning method. The auroral brightness curve extracted from the images is compared with the intensity distribution along the slit until a good match is obtained between the auroral features observed in the image and the spectrum. This position is used to define the intersection of the STIS slit with the auroral oval and, if present, high-latitude spots. In this case, the slit did not cross the Io tail but intercepted the dayside and nightside main oval and high-latitude spots. The location of the different emission features is best seen in a polar projection (Figure 3b). Parallels of latitude and System III (SIII) meridians are marked by the white dots in Figure 3. The main polar spot in the slit is near $78^{\circ} \mathrm{N}$ latitude and $\lambda_{\text {III }}=$ $206^{\circ}$, the System III longitude. At the time of the image exposure, this spot was weaker than the main oval and the limb.

[15] Figure 4a shows the time tag spectral sequence $(630 \mathrm{~s})$ observed at 1221 UT during the same HST orbit. Each photon event on the MAMA detector is represented as an individual point. The limb and main oval emissions are fairly constant in time and location. Three polar emission 


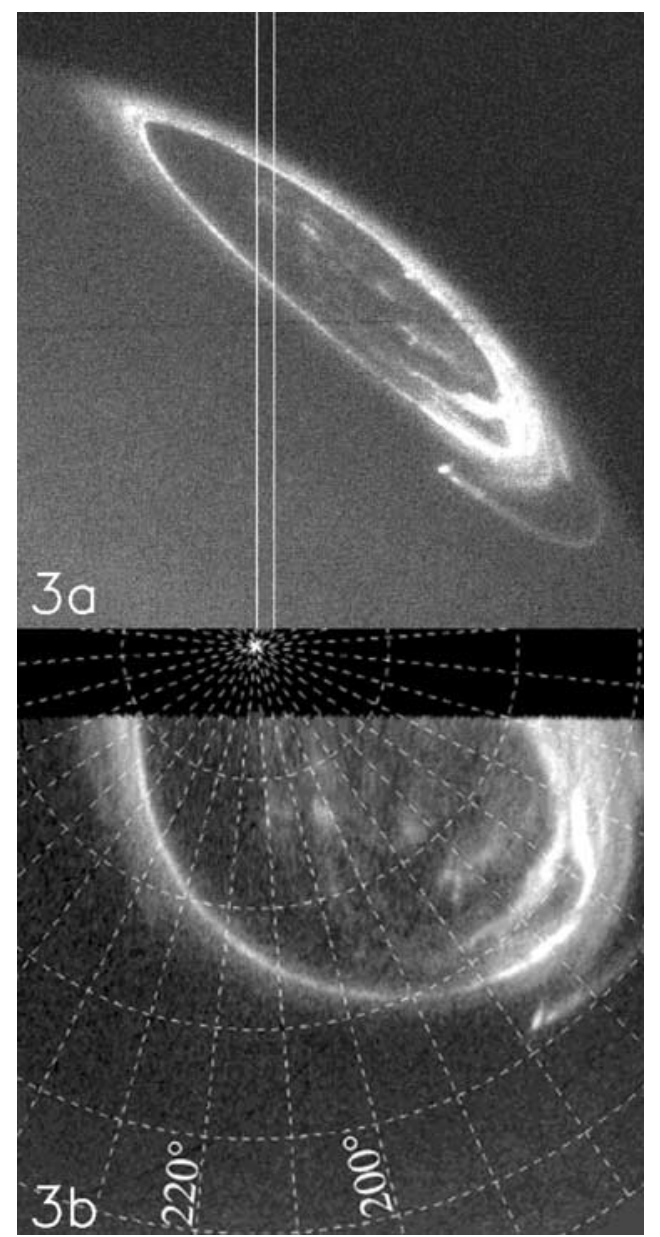

Figure 3. (a) Raw image obtained on 14 December 2000 at 1213 UT with the Space Telescope Imaging Spectrograph MultiAnode Microchannel Array (STIS MAMA) aperture. The main regions of the Jovian aurora, limb aurora, highlatitude emission, main oval, and Io tail, are easily identified. The position of the STIS slit used to obtain spatially resolved spectroscopy of the Jovian aurora $8 \mathrm{~min}$ later is marked by the double line crossing the polar region. (b) Polar projection of the same image showing the Io trail, the double oval eastward of $180^{\circ}$, and the polar spots. The magnetic pole is indicated by the white dot at the intersection of the meridians. Magnetic parallels and meridians are separated by $10^{\circ}$. The polar spot crossed by the slit visible in Figure $3 \mathrm{a}$ is presumably seen at $66^{\circ} \mathrm{N}$, $174^{\circ} \mathrm{SIII}$.

intensifications occurred after $40 \mathrm{~s}, 360 \mathrm{~s}$, and during the last $50 \mathrm{~s}$ of the exposure. Figures $4 \mathrm{~b}$ and $4 \mathrm{c}$ show the unabsorbed intensity longward of $1500 \AA$ and the color ratio, both averaged over 10-s segments and smoothed over five data points. Error bars in Figure $4 \mathrm{~b}$ correspond to the 1- $\sigma$ standard deviation associated with the 10-s average count rates. The bars in Figures $4 \mathrm{c}$ and $4 \mathrm{~d}$ are obtained by converting the statistical error on the count rate of both spectral regions defining the color ratio into an uncertainty on the color ratio and the average electron energy, based on values shown in Figure 2. The $\mathrm{H}_{2}$ emission rate associated with the peaks of the three brightenings are 32,43 , and
$55 \mathrm{kR}$, respectively, corresponding to a precipitated energy flux of $4.7,6.3$, and $7.0 \mathrm{~mW} \mathrm{~m}^{-2}$. They indicate that the first $200 \mathrm{~s}$ and last $100 \mathrm{~s}$ present a fair amount of correlation between the electron energy flux and the color ratio during the first $250 \mathrm{~s}$, although the latter remains close to $\sim 2$. To illustrate the correlation between the precipitated flux and the electron mean energy in further details, Figure $4 d$ shows the same event with the color ratio converted into mean electron energy as explained before. In parallel with the intensification at $360 \mathrm{~s}$, the mean energy decreases from 60 to $45 \mathrm{keV}$. It should be noted that the error bars on the color ratio are relatively large, especially during periods of low count rate.

[16] Another example of a time tag observation is shown in Figures 5 and 6 It was obtained on 20 January 2001 following the 108-s image exposure observed at 1550 UT and shown in Figure 5a. A $9.6^{\circ}$ zone along the dawn limb is in darkness while the rest of the Jovian disk is sunlit. The STIS slit crossed the Io trailing tail, the dayside main oval, dayside high-latitude spots, and the nightside main oval as traced by the slit projection in Figure 5a. A bright spot inside the main oval is conspicuous in the polar projection (Figure 5b). Its center is located at $61^{\circ} \mathrm{N}$ and $\lambda_{\text {III }}=169^{\circ}$ but was apparently not crossed by the STIS slit. Other dimmer and more diffuse emissions are observed at high latitudes, filling most of the region inside the auroral oval. Figure $6 \mathrm{a}$ shows the 230-s spectral time tag sequence observed with the $52 \times 0.5$ arc $\sec ^{2}$ aperture, taken $18 \mathrm{~min}$ after the image. The various regions intercepted by the slit are indicated by arrows. The main oval and the Io tail show no significant time variation. Two different regions are considered inside the main oval. Region 1 is initially part of a diffuse auroral emission with no latitudinal structure extending between the dayside oval and nightside limb. An intensification occurred at $t=100 \mathrm{~s}$, reached a peak soon after $150 \mathrm{~s}$, and almost disappeared before the end of the exposure. Region 2 is located poleward of region 1 and shows distinct differences in its time evolution. Its brightening started earlier and shows more temporal structure. This spectrum was preceded by other ones $10 \mathrm{~min}$ earlier and $10 \mathrm{~min}$ later, respectively. They did not exhibit any significant time variation.

[17] Figures $6 b-6 g$ show the unabsorbed intensity, color ratio, and electron energy for regions 1 and 2. The auroral intensity (energy flux) increased by nearly a factor of 5 during the single transient brightening in region 1 (Figure 6b), reaching a maximum of $\sim 195 \mathrm{kR}\left(28 \mathrm{~mW} \mathrm{~m}^{-2} \mathrm{~s}^{-1}\right)$ after $160 \mathrm{~s}$. The color ratio remained constant during the first $120 \mathrm{~s}$ and slightly decreased during the period of most intense brightening. The electron energy, initially close to $50 \mathrm{keV}$, was anticorrelated with the time variation of the precipitated energy flux until the end of the exposure. In region 2 (Figures 6e, and 6f, and 6g) the intensity shows a double peak. A generally positive correlation between the energy flux and $\langle E\rangle$ was observed during the first $110 \mathrm{~s}$, but the two quantities decorrelate soon after. The electron energy reached $120 \mathrm{keV}$, in phase with the first brightness peak at $110 \mathrm{~s}$. The brightness rise and drop times in region 1 is on the order of $100 \mathrm{~s}$. The risetime is slightly longer in region 2. The peak brightness was $310 \mathrm{kR}\left(45 \mathrm{~mW} \mathrm{~m}^{-2} \mathrm{~s}^{-1}\right)$.

[18] A 700-s sequence of the south region (4 July 1997, start time 0932 UT) is displayed in Figures $7 a-7 d$. No FUV image close in time to the time tag spectrum was available 
on this day. The main oval shows a continuous poleward drift with a progressive thinning and fading (Figure 7a). At the same time its color ratio remains approximately constant at $\sim 2$ (not shown). High-latitude brightenings are observed after $100 \mathrm{~s}$ and $230 \mathrm{~s}$ and during the last $70 \mathrm{~s}$ of the exposure. Figure $7 \mathrm{~b}$ indicates that the unabsorbed intensity increases by a factor of 2 in $\sim 50 \mathrm{~s}, 35 \mathrm{~s}$, and $50 \mathrm{~s}$, respectively. The drop time of the first two brightenings are also in the 40- to 50-s range. The total $\mathrm{H}_{2}$ emission rate of the two brightenings is $\sim 105 \mathrm{kR}$, i.e., an electron energy
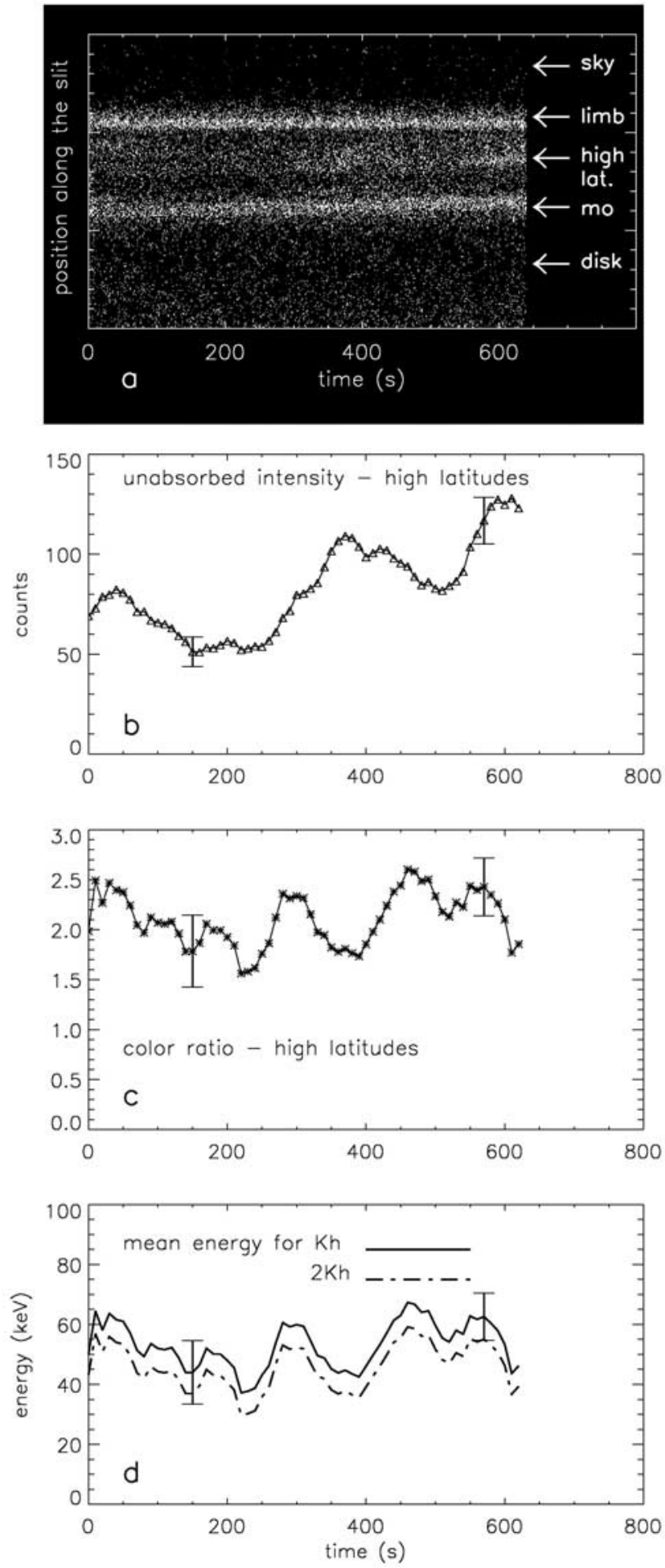

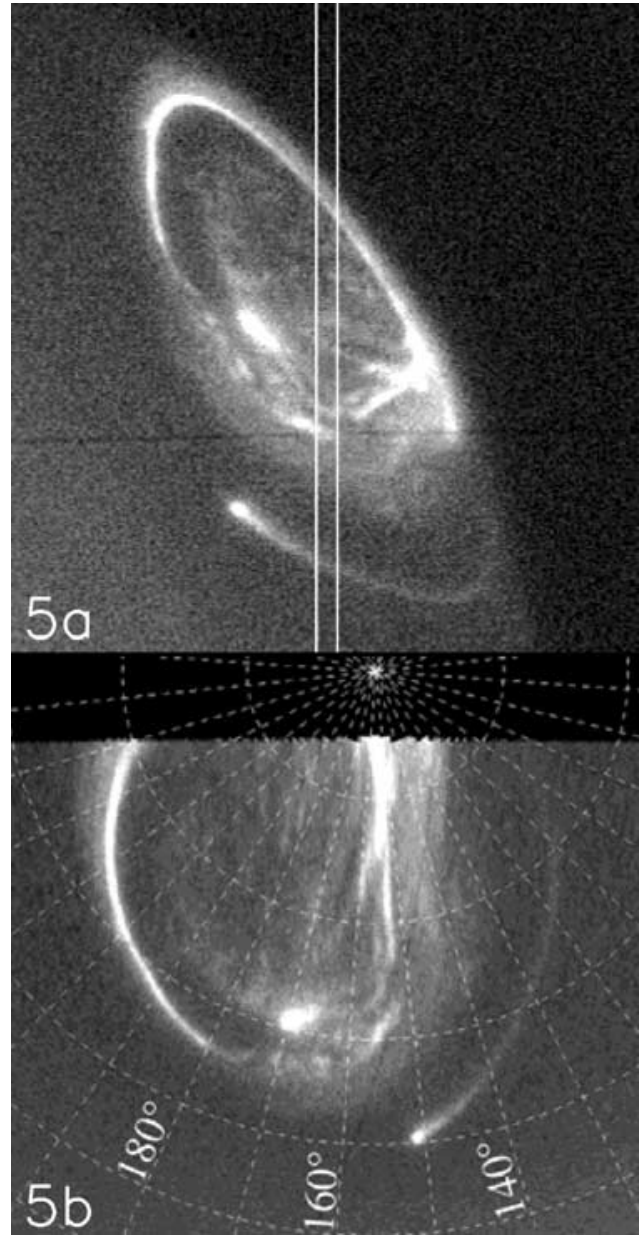

Figure 5. (a) Raw STIS image obtained on 20 January 2001 at 1550 UT with the STIS MAMA CLEAR aperture. The position of the STIS slit used to obtain spatially resolved spectroscopy of the Jovian aurora 18 min later is marked by the solid double vertical line crossing the polar region. (b) Polar projection of the same image showing the Io trail, the main oval, and the polar emission.

flux of $15 \mathrm{~mW} \mathrm{~m}^{-2}$. The color ratio shows little variation during the first $250 \mathrm{~s}$. A minimum CR of 2 corresponds to the first intensity enhancement while the 180-s intensity dip is anticorrelated with the CR. Similarly, the 330-s intensity

Figure 4. (opposite) Time variations observed in the spectral mode from 1221 UT on Dec. 14, 2000 with the $52 \times 0.5 \operatorname{arc~sec}^{2}$ aperture. (a) Time variation of the count rate integrated longward of $1500 \AA$ measured along the STIS slit. The data were averaged over 10-s segments and smoothed over five data points. The main latitudinal structures of emission can be identified with features observed in Figure 3a. (b) Integrated count rate of unabsorbed $\mathrm{H}_{2}$ emission (1500-1700 A) in the high-latitude emission region. The error bars indicate the statistical noise (1 standard deviation). (c) Color ratio for the same region as Figure 4b. (d) Variation of the electron mean energy derived from the color ratio using $K_{H}=1.4 \times 10^{6} \mathrm{~cm}^{2} \mathrm{~s}^{-1}$ at the homopause (denoted by solid line) and twice this value (denoted by dotted line). 

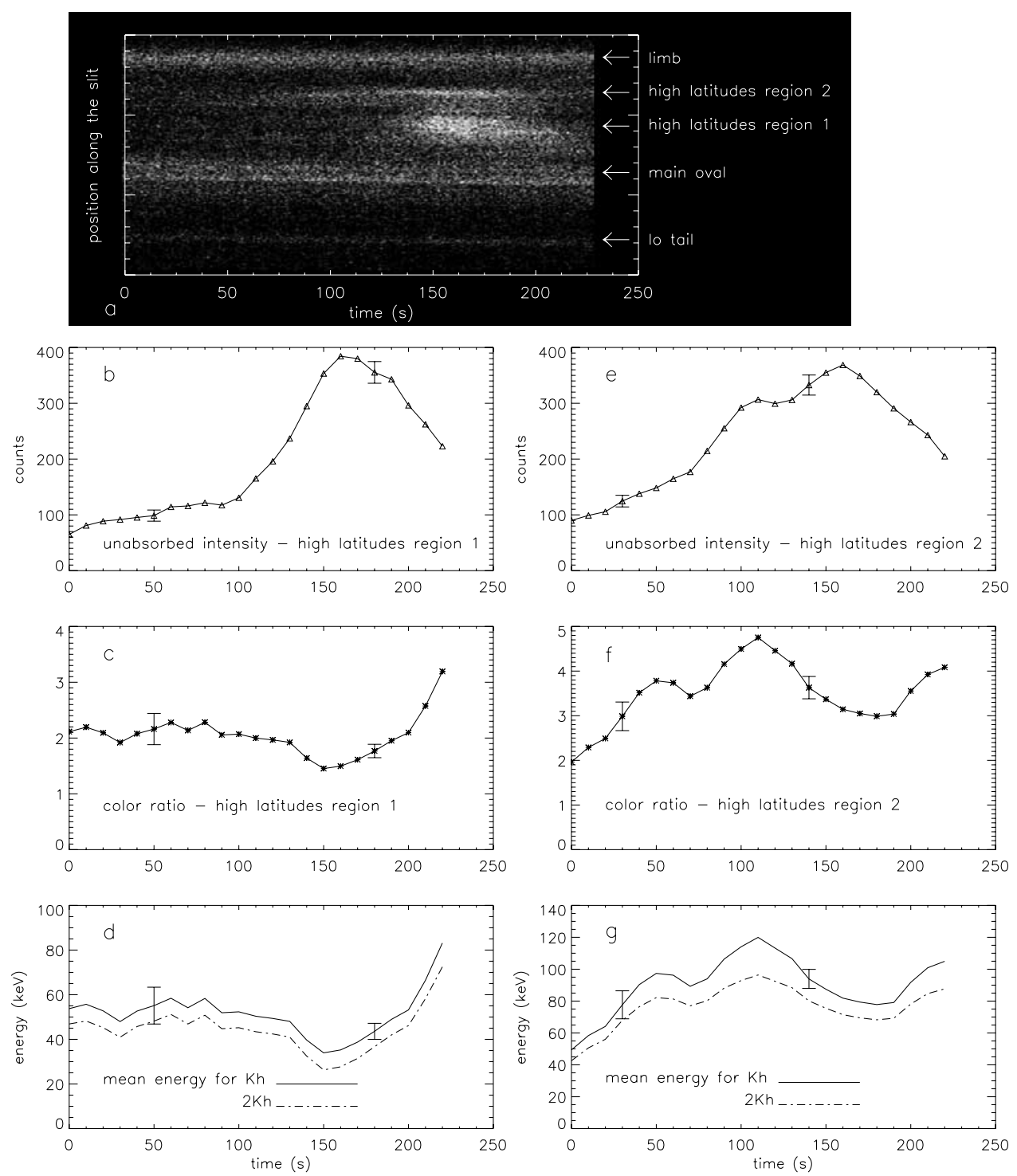

Figure 6. Time variations observed in the spectral mode from 1608 UT on 20 January 2001 with the $52 \times 0.5 \mathrm{arc} \mathrm{sec}^{2}$ aperture. (a) Time variation of the count rate integrated longward of $1500 \AA$ measured along the STIS slit. The main latitudinal structures of emission can be identified with features observed in Figure 5a. (b) Unabsorbed $\mathrm{H}_{2}$ emission in the high-latitude emission region 1. The data were averaged over 10-s segments and smoothed over three data points. The error bars indicate the statistical noise (1 standard deviation). (c) Color ratio for the same region as Figure 6b. (d) Variation of the electron mean energy derived from the color ratio using $K_{H}=1.4 \times 10^{6} \mathrm{~cm}^{2} \mathrm{~s}^{-1}$ at the homopause (denoted by solid line) and twice this value (denoted by dotted line). (e) Integrated count rate of unabsorbed $\mathrm{H}_{2}$ emission in the high-latitude emission region 2. (f) Color ratio for the same region as Figure 6e. (g) Variation of the electron mean energy derived from the color ratio.

minimum is concurrent with a CR maximum of 3.2 , and the brightness rise after $t=500 \mathrm{~s}$ is also parallel to a drop of the color ratio. Figure $7 \mathrm{c}$ shows first a lack of correlation followed by an anticorrelation between the high-latitude electron energy flux and the mean energy.

[19] The fourth case, shown in Figure 8a, displays the variation of the total unabsorbed FUV brightness during a long $(1400 \mathrm{~s})$ observation sequence with the $52 \times 0.2$ arc $\sec ^{2}$ slit. It started at 0947 UT on 4 July 1997, 4 min after the end of the previous one. The slit intercepted the main oval in the noon sector. The SIII longitude of the CML changed from $63^{\circ}$ to $77^{\circ}$ during the observing sequence. Two main regions are readily identified along the STIS slit. The main oval is initially double and both arcs exhibit a slow poleward drift until they merge into a single enhanced arc after $\sim 1000 \mathrm{~s}$. The poleward motion intensifies during the last $300 \mathrm{~s}$ of the exposure. It is likely a consequence of the poleward displacement of the auroral oval during the exposure as the CML longitude changes by $14^{\circ}$ due to the tilt of the magnetic axis. A second region poleward of the oval presents interesting features. A series of intensifications are seen over an extended latitudinal region. The 

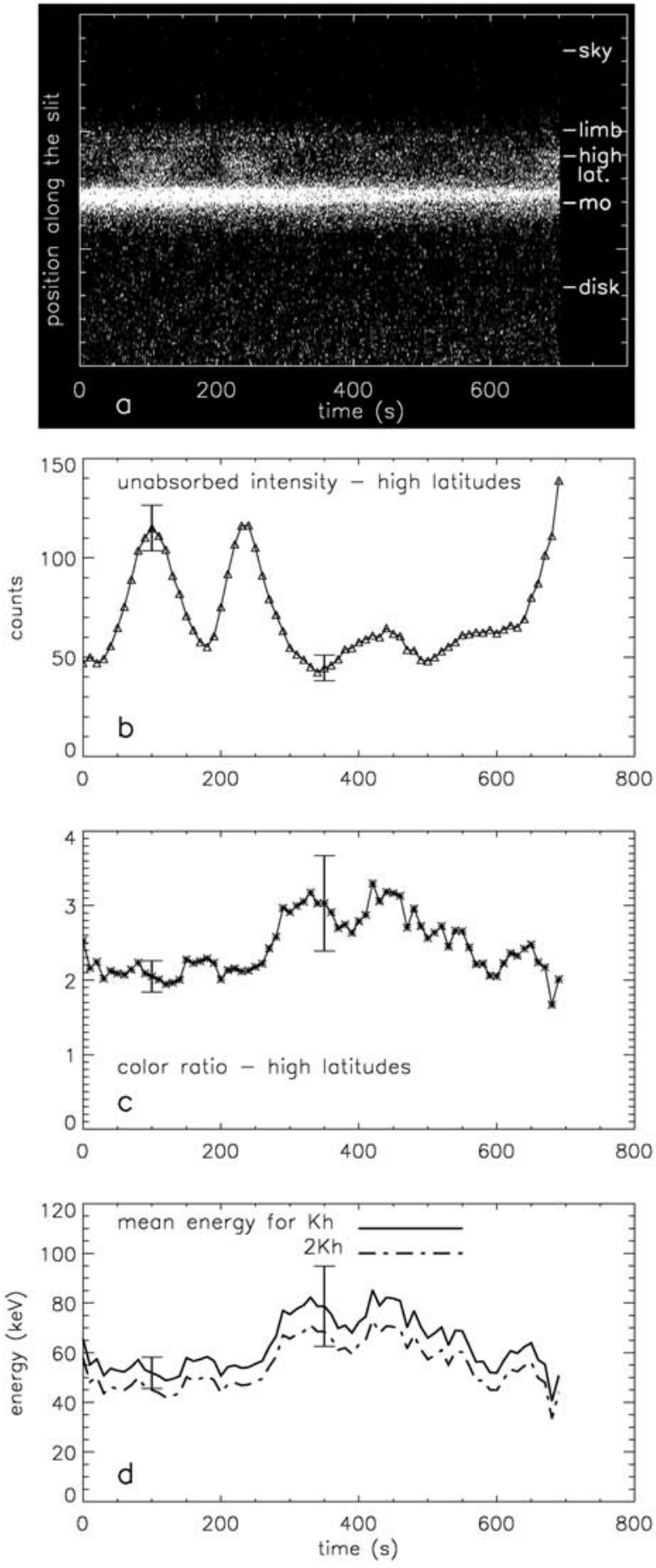

Figure 7. Same as Figure 4 for the spectrum obtained in the south polar region on 4 July 1997 from 0932 UT. (a) Time variation of the count rate integrated longward of $1500 \AA$ measured along the STIS slit. The data were averaged over $10 \mathrm{~s}$ segments and smoothed over five data points. (b) Integrated count rate of unabsorbed $\mathrm{H}_{2}$ emission (1500-1700 $\AA$ ) in the high-latitude emission region. The error bars indicate the statistical noise (1 standard deviation). (c) Color ratio for same region as Figure 7b. (d) Variation of the electron mean energy derived from the color ratio using $K_{H}=1.4 \times 10^{6} \mathrm{~cm}^{2} \mathrm{~s}^{-1}$ at the homopause (solid line) and twice this value (dash-dotted line). strongest bright spot observed after $800 \mathrm{~s}$ appears to initiate at high latitudes and propagate equatorward after $\sim 200 \mathrm{~s}$. At this time its brightness exceeds that of the main oval.

[20] Figures $8 \mathrm{~b}$ and $8 \mathrm{c}$ show the latitudinally integrated time response of the unabsorbed intensity and the FUV color ratio during the same period in the main oval. The main oval shows little brightness change during the first $1000 \mathrm{~s}$. The corresponding CR decreases from 3.2 to 1.5 . The intensity rise observed in Figure 8a following the merging of the two arcs is clearly accompanied by an increase of the CR from $\sim 1.5$ to 3 in 300 s. Figure $8 d$ indicates that $\langle\mathrm{E}\rangle$ dropped from $\sim 90$ to $40 \mathrm{keV}$ during the first $950 \mathrm{~s}$ with fluctuations correlated with the precipitated flux. The mean energy climbed back up to $80 \mathrm{keV} \sim 40 \mathrm{~s}$ before the brightness peak. The correlation between the brightness and the electron mean energy suggests that the intensification and poleward motion of the main oval are closely related to the hardening of the auroral precipitation. The risetimes of the intensity and CR after 930 s are clearly different. The fastest rise of the intensity indicates that both the energy and the precipitated electron number flux increased during this interval.

[21] Figures $8 \mathrm{e}, 8 \mathrm{f}$, and $8 \mathrm{~g}$ show equivalent plots for the high-latitude region. The unabsorbed brightness curve bears the signature of the pulses in Figure $8 \mathrm{a}$. The intensity (and thus the electron energy flux) increases by a factor of 2-3 at the peak of the two flares. Other less intense enhancements are also present. The first three peaks are separated by $\sim 80 \mathrm{~s}$, followed by a dip. The major peak at $850 \mathrm{~s}$ is followed by a secondary enhancement $\sim 150 \mathrm{~s}$ later. It reaches a peak of $190 \mathrm{kR}$ of $\mathrm{H}_{2}$ emission $\left(28 \mathrm{~mW} \mathrm{~m}^{-2}\right)$. No significant variation of the CR is associated with the flares. The apparent modulation of the CR and energies are mainly due to statistics of the count rate as indicated by the error bars. The mean energies range from 35 to over $100 \mathrm{keV}$ in the main oval and $\sim 60 \mathrm{keV}$ in the polar spots. Time integration of the unabsorbed intensity over the length of the main light pulse indicates that a total energy of $1.7 \times 10^{13} \mathrm{~J}$ was carried by the electrons during this event in the $600 \times$ $12,000 \mathrm{~km}^{2}$ intercepted by the slit on the planet.

[22] The four cases described were selected because they show time variations with statistically significant count rates. A survey of the STIS spectra collected with the slit intercepting the high-latitude regions provides a global view of the importance of auroral variations on timescales of a few minutes or less. It may be summarized as follows: (1) The main oval time tag spectra usually show no fast brightness and color ratio changes over periods of a few minutes (not shown in this study), (2) among the 24 highlatitude time tag sequences studied, only 8 show highlatitude brightness changes exceeding $50 \%$, as illustrated in Figures 4b, 6b, 6e, 7b, and 8e, (3) the slower brightness variations are usually positively correlated with the electron mean energy, i.e., an increased precipitated energy flux is accompanied by a progressive hardening of the electron energy spectrum (first $270 \mathrm{~s}$ of Figures $4 \mathrm{~b}-4 \mathrm{c}$ and the first $110 \mathrm{~s}$ of Figures $6 \mathrm{e}-6 \mathrm{f}$ ), and (4) rapid (typically $\sim 100 \mathrm{~s}$ ) intensity changes typically show either no correlation or anticorrelation with the electron mean energy (Figures 6b$6 c$, last $100 \mathrm{~s}$ of Figures $6 \mathrm{e}-6 \mathrm{f}$, Figures $7 \mathrm{~b}-7 \mathrm{c}$, and Figures $8 \mathrm{e}-8 \mathrm{f}$ ). Any of the three situations (correlation, lack of 

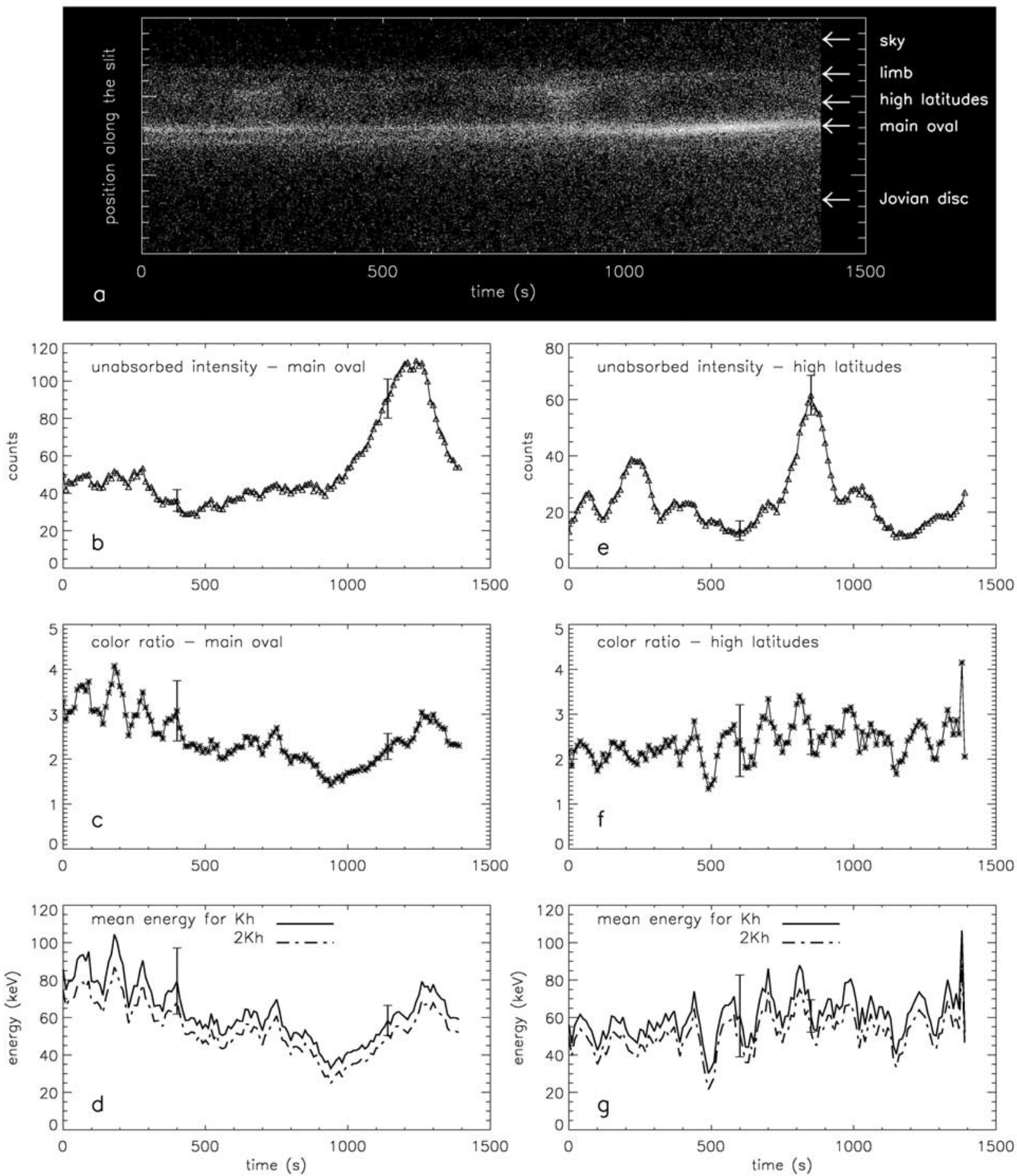

Figure 8. Same as Figure 5 for (a) 4 July 1997 from 0947 UT. (b, c, and d) Main oval. (e, f, and g) High-latitude auroral emission.

correlation, anticorrelation) may be found in a given time sequence.

\section{Discussion}

[23] A first conclusion based on examination of a 3-year data set is the lack of correlation between the brightness changes in the main oval and those in high-latitude spots.
The lack of spatial continuity between the two regions and of simultaneous time response strongly suggests that the source regions in the magnetosphere are decoupled and that the energization processes are different. The electrons precipitating in the main oval are presumably accelerated by upward field-aligned electric fields associated with departure from rigid corotation beyond $\sim 20-30 R_{J}$ [Cowley and Bunce, 2001; Hill, 2001]. The alternative interpretation 
by Frank and Paterson [2002] based on the PLS electron measurements on Galileo is that the main oval aurora is excited by field-aligned electron beams energized by plasma heating in the vicinity of the ionosphere. On the other hand, they suggest that parallel electric fields play a role in the generation of the polar emission inside the main auroral ring.

[24] The time-variable polar spots described in this study are characterized by typical risetime on the order of 1-2 min. STIS spectra alone do not permit one to determine whether the observed changes are spatial or temporal. However, the similarities between these features and the spot brightenings observed in the imaging mode and with the Galileo and Cassini FUV spectrometers strongly suggest that we measure real-time variations rather than a motion of stable auroral features. Even when they positively correlate with the intensity, the time variation of the color ratio does not show any evidence for an early rise of the electron energy preceding the peak of the brightness enhancement. Consequently, these polar flares do not bear the signature of velocity dispersion for a source at large distances such as the equatorial plasma sheet. During some of these transients, no energy increase or even energy drops are associated with polar brightenings. The mean electron energy is generally in the range $40-80 \mathrm{keV}$, comparable to or slightly less than in the main oval (Figures $6 \mathrm{~d}$ and $6 \mathrm{~g}$ ) but higher than in the Io auroral tail [Gérard et al., 2002]. The observations of December 1997 indicate that several transient events took place over a $30-\mathrm{min}$ period in the same longitude sector (the slit was not moved relative to the central meridian between the two exposures). A similar situation occurred with the imaging observation of the polar flares described by Waite et al. [2001], who measured several successive enhancements over a 2-hour period.

[25] Similarities and differences exist between our observations and the polar flares described by Waite et al. [2001]. The two polar flares reported in the literature are considerably brighter than the intensification described in this study. Values as high as 37 MegaRayleighs (MR; $1 \mathrm{MR}=$ $10^{9}$ photon $\mathrm{cm}^{-2} \mathrm{~s}^{-1}$ ), corresponding to an electron energy flux of $\sim 4 \mathrm{Wm}^{-2}$ were observed at the peak of the event. This is significantly higher than the $30-190 \mathrm{kR}$ maximum observed in the spectral mode. Similarly, the 21 September 1999 flare increased by a factor of 30 within $70 \mathrm{~s}$, to be compared with our factor $\sim 2-5$ enhancements reported here. Part of the differences may be real, but it is also likely that the STIS slit did not necessarily catch the location of the largest flaring. In addition, the intensities in the spectral mode are averaged over the width of the STIS slit (20 pixels), in contrast to the 6-pixel resolution of the images. These differences tend to smooth the apparent intensity changes.

[26] Gurnett et al. [2002] describe radio observations from the high-latitude Jovian aurora and their relationship with the interplanetary shocks and the Cassini Ultraviolet Imaging Spectograph Subsystem measurements of auroral brightness. They report simultaneous enhancements of hectometric emission observed from Cassini and Galileo which are triggered by the arrival at Jupiter of compressed solar plasma. The peak of the hectometric radiation coincided with the maximum brightness of the ultraviolet auroral emission and occurred near the time of maximum solar wind plasma density, after correcting for the solar wind travel time. The hectometric radio emissions are produced by the cyclotron maser mechanism [Zarka, 1998] at distances of 1-3 $R_{J}$. At Earth, their counterpart in the kilometric radiation is generated along field lines terminating in bright spots in the aurora but generally not in the polar cap [Huff et al., 1988]. They are colocated with "inverted V" events associated with field-aligned potential drops. The power of the hectometric radiation correlates well with the solar wind dynamic pressure [Desch and Barrow, 1984]. The total auroral infrared brightness [Baron et al., 1996] is also positively correlated with the solar wind ram pressure on timescales of days. A correlation between polar "flares" and solar wind or hectometric radiation on timescales of minutes has not been observed so far.

[27] The transient features described in this study map along field lines from the outer magnetosphere at distances exceeding $\sim 30 R_{J}$. These field lines are probably closely coupled to the dynamical solar wind and may be directly influenced by the magnitude and direction of the interplanetary magnetic field (IMF) [Cowley and Bunce, 2001]. However, no Jovian magnetic field model [Connerney et al., 1998] properly describes the field configuration in these high-latitude regions The transient high-latitude spots are not necessarily the footprints of the daytime cusp region, which is probably closely confined near local noon, in contrast with the fairly widespread location of the active high-latitude FUV emissions. Recent studies of the Earth's cusp Lyman- $\alpha$ signature [Frey et al., 2002] have shown that it is a limited region whose position and brightness depends on the orientation and magnitude of the IMF. It is likely that the high-latitude Jovian flares are the auroral response to the generation of compressions on the front of the magnetosphere in response to abrupt solar wind dynamical pressure rises, analogous to the shock-induced dayside auroral response on Earth [Zhou and Tsurutani, 1999; Hubert et al., 2003]. In situ measurements and MHD simulations of the Jovian magnetosphere [Ogino et al., 1998] show that it rapidly and strongly responds to changes in solar wind dynamic pressure.

[28] Although the exact process remains unclear, we interpret the quasi-constant electron energy associated with several of the polar intensifications as evidence that the precipitation mechanism is not the buildup of an electric field along the field lines mapping to the brightening spot. Instead, it reflects an increase of the number flux of electrons inside the loss cone without any further energization. A possibility is the "magnetic pumping" process [Goertz, 1978] in which a fraction of the increase in perpendicular energy of trapped particles due to the increase of the local magnetic field strength following magnetospheric compression is converted into parallel energy by pitch angle scattering before the magnetic field relaxes to its original configuration. Some of the particles see a lowering of the altitude of their mirror point into the atmosphere so that they lose their kinetic energy in inelatic collisions in the Jovian atmosphere. Pitch angle scattering into the loss cone by Alfvén wave saturation has also been suggested [Zhou and Tsurutani, 1999; Zhou et al., 2003] as a mechanism for the pressure pulse induced intensifications observed on the Earth's dayside. It is possible that a similar process acts in the Jovian outer magnetosphere and enhances the mecha- 
nism that scatters preexisting electrons into the loss cone with little or no change of their characteristic energy. Whether the same mechanism explains the pulsating X-ray hot spot, the FUV polar intensifications and flares, and the infrared polar brightenings is still unclear. It is likely that the enhanced methane infrared emission is caused by increased energetic precipitation below the homopause possibly linked to the source of X-ray emission. Instead, the FUV polar brightenings are not associated with very energetic electron precipitation that would be required if the X-ray emission is produced by bremsstrahlung.

[29] Acknowledgments. This work is based on observations with the NASA/ESA Hubble Space Telescope, obtained at the Space Telescope Science Institute (STScI), which is operated by AURA, Inc., for NASA. J.C.G. and D.G. acknowledge support from the Belgian Fund for Scientific Research (FNRS). The PRODEX program of ESA provided financial support for this research to the University of Liège. This research was also funded by grant GO-08657-01-A from the Space Telescope Science Institute and from NASA to the University of Michigan.

[30] Arthur Richmond thanks Joseph Ajello and another reviewer for their assistance in evaluating this manuscript.

\section{References}

Ajello, J. M., et al., Galileo orbiter ultraviolet observations of Jupiter aurora, J. Geophys Res., 103, 20,125, 1998.

Ballester, G. E., et al., Time-resolved observations of Jupiter's far-ultraviolet aurora, Science, 274, 409, 1996.

Baron, R. L., T. Owen, J. E. P. Connerney, T. Satoh, and J. Harrington, Solar wind control of Jupiter's $\mathrm{H}_{3}^{+}$aurorae, Icarus, 120, 437, 1996.

Bunce, E. J., and S. W. H. Cowley, Divergence of the equatorial current in the dawn sector of Jupiter's magnetosphere: Analysis of Pioneer and Voyager magnetic field data, Planet. Space Sci., 49, 1089, 2001

Clarke, J. T., Far-UV imaging of Jupiter's aurora with HST/WFPC 2, Science, 274, 404, 1996.

Clarke, J. T., et al., Hubble Space Telescope imaging of Jupiter's UV aurora during the Galileo orbiter mission, J. Geophys. Res., 103, 20,217, 1998

Connerney, J. E. P., M. H. Acuña, N. F. Ness, and T. Satoh, New models of Jupiter's magnetic field constrained by the Io flux tube footprint, J. Geophys. Res., 103, 11,929, 1998.

Cowley, S. W. H., and E. J. Bunce, Origin of the main auroral oval in Jupiter's coupled magnetosphere-ionosphere system, Planet. Space Sci., 49, 1067, 2001

Desch, M. D., and C. H. Barrow, Direct evidence for solar wind control of Jupiter's hectometer-wavelength radio emission, J. Geophys Res., 89, $6819,1984$.

Dols, V., J. C. Gérard, J. T. Clarke, J. Gustin, and D. Grodent, Diagnostics of the Jovian aurora deduced from ultraviolet spectroscopy: Model and GHRS observations, Icarus, 147, 251, 2000.

Drossart, P., B. Bézard, S. K. Atreya, J. Bishop, J. H. Waite Jr., and D. Boice, Thermal profiles in the auroral regions of Jupiter, J. Geophys. Res., 98, 18,803, 1993.

Frank, L. A., and W. R. Paterson, Galileo observations of electron beams and thermal ions in Jupiter's magnetosphere and their relationship to the auroras, J. Geophys. Res., 107(A12), 1478, doi:10.1029/2001JA009150, 2002.

Frey, H. U., S. B. Mende, T. J. Immel, S. A. Fuselier, E. S. Claflin, J. C. Gérard, and B. Hubert, Proton aurora in the cusp, J. Geophys. Res. 107(A7), 1091, doi:10.1029/2001JA900161, 2002.

Gérard, J.-C., and V. Singh, A model of energetic electrons and EUV emission in the Jovian and Saturnian atmospheres and implications, J. Geophys. Res., 87, 4525, 1982.

Gérard, J.-C., V. Dols, R. Prangé, and F. Paresce, The morphology of the north Jovian ultraviolet aurora observed with the Hubble Space Telescope, Planet. Space Sci., 42, 905, 1994.

Gérard, J.-C., J. Gustin, D. Grodent, P. Delamere, and J. T. Clarke, Excitation of the FUV Io tail on Jupiter: Characterization of the electron precipitation, J. Geophys. Res., 107(A11), 1394, doi:10.1029/2002JA009410, 2002.

Gladstone, G. R., M. Allen, and Y. L. Yung, Hydrocarbon photochemistry in the upper atmosphere of Jupiter, Icarus, 119, 1, 1996.

Gladstone, G. R., et al., A pulsating auroral X-ray hot spot on Jupiter, Nature, 415, 1000, 2002
Goertz, C. K., Energization of charged particles in Jupiter's outer magnetosphere, J. Geophys. Res., 83, 3145, 1978.

Grodent, D., G. R. Gladstone, J. C. Gérard, V. Dols, and J. H. Waite, Simulation of the morphology of the Jovian UV North aurora observed with the Hubble Space Telescope, Icarus, 128, 306, 1997.

Grodent, D., J. H. Waite Jr., and J.-C. Gérard, A self-consistent model of the Jovian auroral thermal structure, J. Geophys. Res., 106, 12,933, 2001.

Grodent, D., J. T. Clarke, J. Kim, J. H. Waite Jr., and S. W. H. Cowley, Jupiter's main auroral oval observed with HST-STIS, J. Geophys. Res., 108, doi:10.1029/2003JA009921, in press, 2003.

Gurnett, D. A., et al., Control of Jupiter's radio emission and aurorae by the solar wind, Nature, 415, 985, 2002.

Gustin, J., D. Grodent, J.-G. Gérard, and J. T. Clarke, Spatially resolved far ultraviolet spectroscopy of the Jovian aurora, Icarus, 157, 91, 2002.

Harris, W. M., J. T. Clarke, M. A. McGrath, and G. E. Ballester, Analysis of Jovian auroral H Lyman- $\alpha$ Emission (1981 - 1990), Icarus, 23, 350, 1996.

Hill, T. W., The Jovian auroral oval, J. Geophys. Res., 106, 8101, 2001.

Hubert, B., J. C. Gérard, S. A. Fuselier, and S. B. Mende, Observation of dayside subauroral proton flashes with the IMAGE-FUV imagers, Geophys. Res. Lett., 30(3), 1145, doi:10.1029/2002GL016464, 2003.

Huff, R. L., W. Calvert, J. D. Craven, L. A. Frank, and D. Gurnett, Mapping of auroral kilometric radiation sources to the aurora, J. Geophys. Res., 93, $11,445,1988$

Kim, Y. H., J. L. Fox, and J. J. Caldwell, Temperatures and altitudes of Jupiter's ultraviolet aurora inferred from GHRS Observations with the Hubble Space Telescope, Icarus, 128, 189, 1997

Livengood, T. A., D. F. Strobel, and H. W. Moos, Long-term study of longitudinal dependence in primary particle precipitation in the north Jovian aurora, J. Geophys. Res., 95, 10,375, 1990.

McKibben, R. C., J. A. Simpson, and M. Zhang, Impulsive bursts of relativistic electrons discovered during Ulysses' traversal of Jupiter's dusk-side magnetosphere, Planet. Space Sci., 41, 1041, 1993.

Morrissey, P. F., P. D. Feldman, J. T. Clarke, B. C. Wolfven, D. F. Strobel, S. T. Durrance, and J. T. Trauger, Simultaneous spectroscopy and imaging of the Jovian aurora with the Hopkins Ultraviolet Telescope and the Hubble Space Telescope, Astrophys. J., 476, L918, 1997.

Ogino, T., R. J. Walker, and M. G. Kivelson, A global model magnetohydrodynamic simulation of the Jovian magnetosphere, J. Geophys. Res., $103,225,1998$

Prangé, R., D. Rego, L. Pallier, J. E. P. Connerney, P. Zarka, and J. Queinnec, Detailed study of FUV Jovian auroral features with the post-COSTAR HST faint object camera, J. Geophys. Res., 103, 20,195, 1998.

Pryor, W. R., A. I. F. Stewart, K. E. Simmons, J. M. Ajello, W. K. Tobiska, J. T. Clarke, and G. R. Gladstone, Detection of rapidly varying emissions in Jupiter's aurora from the Galileo orbiter, Icarus, 151, 314, doi:10.1006/ icar.2001.6604, 2001

Raynaud, E., et al., The 10 October 1999 HIP9369 occultation by the northern polar region of Jupiter: Ingress and egress lightcurves analysis, Icarus, 162, 344, 2003

Trafton, L., J. C. Gérard, G. Munhoven, and J. H. Waite Jr., High-resolution spectra of Jupiter's northern auroral ultraviolet emission with the Hubble Space Telescope, Astrophys. J., 421, 816, 1994.

Waite, J. H., Jr., T. E. Cravens, J. U. Kozyra, A. F. Nagy, S. K. Atreya, and R. H. Chen, Electron precipitation and related aeronomy of the Jovian thermosphere and ionosphere, J. Geophys. Res., 88, 6143, 1983.

Waite, J. H., Jr., et al., An auroral flare at Jupiter, Nature, 410, 787, 2001.

Yung, Y. L., G. R. Gladstone, K. M. Chang, J. M. Ajello, and S. K. Srivastava, $\mathrm{H}_{2}$ fluorescence spectrum from 1200 to $1700 \AA$ by electron impact: Laboratory study and application to Jovian aurora, Astrophys. J. Lett., 254, L65, 1982

Zarka, P., Auroral radio emissions at the outer planets: Observations and theories, Geophys. Res. Lett., 103, 20,159, 1998.

Zhou, X., and B. T. Tsurutani, Rapid intensification and propagation of the dayside aurora: Large-scale interplanetary pressure pulses (fast shocks), Geophys. Res. Lett., 26, 1097, 1999.

Zhou, X.-Y., R. J. Strangeway, P. C. Anderson, D. G. Sibeck, B. T. Tsurutani, G. Haerendel, H. U. Frey, and J. K. Arballo, Shock aurora: FAST and DMSP observations, J. Geophys. Res., 108(A4), 8019, doi:10.1029/ 2002JA009701, 2003.

J. T. Clarke, Department of Astronomy, Center for Space Physics, Boston University, Boston, MA 02215, USA. (jclarke@bu.edu)

J.-C. Gérard, A. Grard, D. Grodent, and J. Gustin, Laboratoire de Physique Atmosphérique et Planétaire, Institut d'Astrophysique et de Géophysique, Université de Liège, B-4000 Liege, Belgium. (jc.gerard@ulg.ac.be; grard@astro.ulg.ac.be; d.grodent@ulg.ac.be; J.Gustin@ulg.ac.be) 